УО «Гродненский государственный медицинский университет», Гродно, Беларусь

Проанализированы и освещчены литературные и собственные данные о возможностях исследования сильы кисти и оценки мелкой моторики кисти у пациентов с иентральными и периферическими парезами.

Ключевые слова: мелкая моторика, кисть, парезы, оценка эффективности медицинской реабилитации

Одним из частых последствий поражения центрального и периферического отделов нервной системы является нарушение двигательной функции в конечностях, что в значительной мере оказывает влияние на самообслуживание и социальное функционирование $[6,4,14]$.

В реабилитации пациентов с центральными и периферическими парезами верхних конечностей важным фактором является степень восстановления функции кисти как наиболее важного органа социальной и профессиональной деятельности, так как пациент в результате данной патологии теряет возможность двуручной работы, которая связана в основном с манипуляцией предметами и множеством социальных и профессиональных функций [2].

В работе верхней конечности основной является функция захвата и удержания предметов, а также скоординированные движения пальцев. Кисть как наиболее подвижная часть руки выполняет как работу, требующую большой силы, так и легкую высокоточную работу, совершая при этом большое количество движений. Для повышения качества жизни восстановление функции кисти является важной задачей [2, 12, 13$]$.

Современные методы медицинской реабилитации (МР) постоянно развиваются, но способы оценки динамики мелкой моторики достаточно ограничены и субъективны, что приводит к затруднению объективной оценки эффективности MP.

Вместе с тем объективная оценка силы мышц кисти у пациентов с центральными и периферическими парезами затруднена по причине того, что зачастую пациент не может выполнить метод измерения в соответствии со стандартом проведения, а методы оценки мелкой моторики у данной категории пациентов недостаточно раскрыты в современной научной и учебной литературе [14].

В настоящий момент распространенными объективными методами измерения функционирования кисти являются углометрия для оценки объема движений в суставах и динамометрия для определения силы мышц [5]. Но результатов данных методов недостаточно для оценки восстановления мелкой моторики, так как, кроме объема движений и мышечной силы, также важен анализ точной направленности скоординированных движений и истощаемость.
Так, известен способ оценки состояния двигательной функции кисти с использованием цифровых камер и светоотражающих маркеров. Оценивается состояние двигательной функции кисти, включающее фиксацию движений кисти с помощью цифровых камер с частотой измерения $120 \mathrm{~Hz}$ и полусферических маркеров со светоотражающим покрытием, при наложении на каждую кисть 23 маркеров [9].

Недостатком данного метода является необходимость использования специального дорогостоящего оборудования и наличия специальных навыков у медперсонала. Объективность результатов исследования по данному методу зависит и от нескольких других показателей, что в свою очередь требует дополнительных затрат времени. Устройство в большей степени предназначено для измерения объема движения в структурах кисти и не может служить для полной оценки состояния мелкой моторики, так как не учитывает силу мышц, способность к удержанию, манипулированию мелкими предметами и истощаемость.

Известен также способ биомеханического исследования кисти, основанный на конструкции, которая содержит вертикальную стойку, укрепленную на основании, шарнирный узел и подпружиненный зажим фаланги, элементы крепления кисти и пальца, элемент передачи движения, на свободном конце которой расположен подпружиненный зажим фаланги с возможностью перемещения в разных плоскостях, поддерживающий столик, вспомогательное ложе, измерительный блок на шарнирном соединении и передающей штанге, измерители изменения расстояния в одной системе координат для каждой регистрирующей пары в виде оптически соединенных источника инфракрасного излучения и фотоприемника [11].

Недостатком данного устройства является его громоздкость, необходимость использования специального оборудования, затраты времени на подготовку и проведение исследования и наличия специальных навыков у медперсонала. Устройство в большей степени предназначено для измерения амплитуды движения в кисти и не учитывает силу мышц, способность к удержанию и манипулированию мелкими предметами и истощаемость. 
Указывается метод развития мелкой моторики кисти и подготовки руки к письму у детей дошкольного возраста, заключающийся в манипулировании 10-15 карандашами или счетными палочками, которые необходимо собрать в кулак по одному предмету, затем также по одному положить на стол. Выполняется без помощи второй руки [1].

Недостатком данного метода, с позиции определения развития мелкой моторики кисти, является неспособность оценить истощаемость, так как отсутствуют повторные этапы и не учитывается количество ошибок; не учитывается время выполнения задания, что ограничивает использование данного метода для объективной оценки восстановления мелкой моторики кисти.

Применяемая в настоящее время в клинике оценка силы кисти по 6-балльной шкале и балльная оценка по Столяровой недостаточно объективны, основаны на субъективных ощущениях врача, вследствие чего могут по-разному трактоваться, не в полной мере отражают дефицит функции, малоинформативны для пациента, не всегда позволяют объективно оценить эффективность медицинской реабилитации и больше подходят для скрининговой оценки нарушения [10].

В связи с этим нами предложена методика, основанная на показателях динамометрии, которая позволит объективизировать результаты реабилитации у пациентов с центральными и периферическими парезами.

Учитывая, что нормы силы кисти у пациентов с парезами не определены, а также то, что на силу кисти влияет ряд факторов, таких как возраст, наличие сопутствующих заболеваний, тренированность пациента, то в соответствии с предложенным методом сила кисти здоровой конечности измеряется кистевым динамометром. Данный показатель является контрольным и принимается за $100 \%$, для удобства и возможности числового выражения 1\% приравнивается к 1 баллу.

Большинство пациентов с центральными и периферическими парезами имеют ограниченный объем движений в суставах, что ведет к невозможности исполнения динамометрии в соответствии со стандартом. Учитывая, что целью динамометрии является оценка развития силы мышц кисти, а предложенная модификация направлена на оценку результатов реабилитационных мероприятий, допускается выполнение динамометрии в положении кисти, доступном пациенту. Учитывая тот факт, что динамометрия до и после курса медицинской реабилитации проводится в одном и том же исходном положении, можно утверждать, что данный способ с учетом особенностей функционирования верхней конечности у пациентов уместно использовать для оценки динамики восстановления силы мышц кисти.

С помощью динамометра определятся сила паретичной конечности, устанавливается выраженность дисфункции в процентном показателе, что позволяет перейти к системе функциональ- ных классов по Стасевичу-Смычку, а процентное соотношение к здоровой конечности переводится в баллы, что позволяет объективно рассчитать силу кисти паретичной конечности по 100-балльной шкале [7].

Например: Пациент Ж., 67 лет, инфаркт в ЛКБ, умеренный правосторонний гемипарез.

Сила здоровой (левой) конечности до курса $\mathrm{MP}-20$ кг/с.

Сила паретичной (правой) конечности до курса МР - 12 кг/с.

Применив предложенную нами методику и приняв силу здоровой конечности $(20$ кг/с) за $100 \%$, можно рассчитать, что сила паретичной конечности (12 кг/с) составляет $60 \%$ от силы здоровой конечности и соответствует 60 баллам по 100-балльной шкале, соответственно, утрата функции составляет $40 \%$, что соответствует II функциональному классу по Стасевичу-Смычку.

После курса МР получили следующие результаты:

Сила здоровой (левой) конечности после курca MP -23 кг/с.

Сила паретичной (правой) конечности после курса МР - 18 кг/с.

Применив ту же систему расчёта, можно отметить, что после проведенного курса МР сила паретичной конечности составляет 78 баллов (78\% от силы здоровой конечности), а, следовательно, утрата функции равняется $22 \%$, что соответствует I функциональному классу. Появляется возможность объективно оценить эффективность МР - прирост силы кисти составил 18 баллов и один функциональный класс.

Для подтверждения эффективности предложенного способа были отобраны пациенты из числа проходивших курс МР в УЗ «Гродненская областная клиническая больница медицинской реабилитации», у которых прирост силы кисти по 6-балльной шкале определялся как 0 балов и являлся статистически не достоверным. Расчет данного показателя по предложенному нами методу показал, что прирост по 100-балльной шкале составил $13,5(11,3 ; 14,0)$, соответственно, медиана прироста силы кисти в процентном соотношении к здоровой составила $13,5 \%$ (табл.). Таким образом, предложенная методика наглядно отражает динамику прироста силы кисти, которая не выявляется при оценке по 6-балльной шкале, а, следовательно, является более чувствительной.

Таблица. - Изменение силы кисти паретичной руки (в баллах) до и после курса реабилитации (Ме (25\%; $75 \%)$

\begin{tabular}{|c|c|c|}
\hline \multirow{2}{*}{$\mathrm{N}=27$} & \multicolumn{2}{|c|}{ Методики } \\
\cline { 2 - 3 } & 6-балльная методика & 100-балльная методика \\
\hline $\begin{array}{c}\text { Прирост } \\
\text { баллов }\end{array}$ & $0(0 ; 0)$ & $13,5(11,3 ; 14,0) *$ \\
\hline
\end{tabular}

Примечание: *-достигнут уровень статистической значимости по сравнению с исходным значением по критерию знаков $(p<0,001)$

Кроме оценки силы кисти и объема движений в суставах важна оценка способности скоординированного захвата, манипулирования, удержания мелких предметов и истощаемость. 
Нами предложен метод, заключающийся в подсчете времени, затрачиваемого обследуемым на манипуляции со счетными палочками, проводимого в 2 этапа:

1. Сбор палочек с последующим удержанием.

2. Удержание с последующим

раскладыванием.

Каждый этап повторяется три раза с 1-минутным перерывом. В процессе каждого этапа задействованы пинцетный, крючковой и щипковый виды захватов, которые являются основой мелкой моторики, а также способность к удержанию мелких предметов в ладони за счет сгибания 2-5 пальцев при участии 1 пальца, оказывающего противопоставление [3].

Регламент проведения метода: 1. Поверхность для проведения метода должна быть гладкая, твердая и ровная. 2. Сбор и раскладывание счетных палочек должно производиться по одной единице. 3. Недопустимо использование особенностей поверхности, на которой разложены палочки (например, угол стола, неровности поверхности). 4. В случае выпадения одной или нескольких удерживаемых палочек в процессе исследования проводимый этап расценивается как ошибка, а результаты не засчитываются, что требует повторного выполнения через 1 минуту. 5. После завершения первого этапа (сбор и последующее удержание) следует второй этап (удержание с последующим раскладыванием). 6. Фиксируется время исполнения каждого этапа. 7. Допустимо неоднократное проведение исследования, но обязательным является обследование по предложенной методике в начале и в конце оказания реабилитационной помощи. 8. Данные, полученные в результате обследования до начала проведения реабилитационных мероприятий, регистрируются, и в дальнейшем используются для сравнения с данными, полученными после завершения реабилитации.

Для проведения обследования рекомендовано использование 10 счетных палочек.

Например: Пациент К., 52 года, анапластическая астроцитома левой затылочной доли головного мозга, состояние после оперативного лечения, умеренный левосторонний гемипарез.

Начальное время, затрачиваемое на манипуляции со счетными палочками, составило 24,5 секунды, допущено 8 ошибок, а также отмечалось увеличение времени при выполнении повторных заданий. При повторном обследовании

\section{Литература}

1. Александрова, А. Опыт работы по развитию мелкой моторики у детей с ОНР [Электронный ресурс] / A. Александрова // Дошкольник : сайт для всей семьи. - Режим доступа: http://doshkolnik.ru/motorika/10785opyt-raboty.html. - Дата доступа: 12.03.2017.

2. Аретинский, В. Б. Восстановление двигательной функции кисти у больных с инсультом с использованием системы "Hand tutor" / В. Б. Аретинский, Е. В. Телегина, Л. И. Волкова // Уральский медицинский журнал. - 2014. - № 9 (123). - С. 46-49. через 7 суток было выявлено, что время, затрачиваемое пациентом на манипуляции со счетными палочками, уменьшилось и составило 23 секунды, что соответствует улучшению мелкой моторики кисти от исходного состояния на $6,13 \%$ и 19,3 сек. через две недели, что соответствует улучшению мелкой моторики кисти от исходного состояния, улучшение на 21,23\%. Также отмечалось стабильное сокращение разницы временных показателей при выполнении последующих заданий и уменьшение количества ошибок, что свидетельствует о снижении истощаемости.

Таким образом, учитывая безусловную необходимость дальнейшего развития и совершенствования системы медицинской реабилитации, предложенные методы являются значимым дополнением к стандартным способам оценки результатов проведенных реабилитационных мероприятий у пациентов с двигательными дисфункциями вследствие патологии нервной системы, что в свою очередь положительно отразится на качестве проведения медицинской реабилитации и мотивации пациента на дальнейшее восстановление.

\section{Выводы}

1. Отдельные современные методы оценки функционирования кисти в процессе медицинской реабилитации недостаточно объективны или требуют значительных материальных затрат и обучения медицинского персонала.

2. Предложенный метод оценки силы кисти предусматривает объективную инструментальную и относительную оценку в сравнении с кистью здоровой конечности. Применение метода дает возможность определить исходный функциональный класс по Стасевичу-Смычеку и оценить эффективность медицинской реабилитации в том числе в пределах функционального класса.

3. Предложенный метод оценки динамики восстановления мелкой моторики кисти позволяет объективно исследовать восстановление скоординированных движений кисти, способность к захвату, манипулированию, удержанию мелких предметов и показатель истощаемости.

4. Предлагаемые методы также позволяют пациенту самостоятельно сравнивать результаты оценки восстановления функций кисти, что, безусловно, положительно влияет на мотивацию к дальнейшему активному участию в реабилитационном процессе.

3. Волкова, А. М. Хирургия кисти : в 3 т. / А. М. Волкова. - Екатеринбург : Уральский рабочий, 1991. - Том 1 : Хирургия сухожилий и нервов кисти. -304 с.

4. Опыт применения роботизированной механотерапии в реабилитации детей с двигательными нарушениями различного генеза / Ю. В. Лобзин [и др.] // Медицина экстремальных ситуаций. - 2015. - № 1 (51). - С. 2226.

5. Пирогова, Л. А. Основы медицинской реабилитации и немедикаментозной терапии : учебное пособие / Л. А. Пирогова. - Гродно : ГрГМУ, 2008. - 212 с. 
6. Роль пластичности головного мозга в функциональной адаптации организма при детском церебральном параличе с поражением рук / А. А. Баранов [и др.] // Педиатрическая фармакология. - 2012. - Том 9, № 6 . - C. 24-32.

7. Основы медицинской реабилитации пациентов, перенесших мозговой инсульт / В. Б. Смычек [и др.] // Неврология и нейрохирургия. Восточная Европа. 2011. - № 3. - C. 24-39.

8. Создание, развитие и современное состояние службы медико-социальной экспертизы и реабилитации в Республике Беларусь / В. Б. Смычек [и др.] // Медицинский журнал. - 2004. - № 1 (7). - С. 4-6.

9. Способ оценки состояния двигательной функции кисти : патент RU 2371088 / О. А. Шавловская. - Опубл. 27.10.2009. - $12 \mathrm{c}$.

10. Столярова, Л. Г. Реабилитация больных с постинсультными двигательными расстройствами / Л. Г. Столярова, Г. Р. Ткачева. - Москва : Медицина, 1978. $-216 \mathrm{c}$.

11. Устройство для биомеханического исследования кисти : патент RU 2066116 / А. В. Кодин, С. Е. Львов, Н. А. Коробов, Н. А. Румянцев, Д. А. Миронов. - Опубл. 10.09.1996.

12. Физическая реабилитация пациентов с ампутационными дефектами верхних конечностей в период обучения пользования протезами / М. А. Борисов [и др.] // Слобожанський науково-спортивний вісник. - 2011. - № 2 (26). - С. 169-175.

13. Цымбалюк, В. И. Селективная фасцикулотомия и нейростимуляция в лечении спастичности кисти / В. И. Цымбалюк, Ю. А. Зозуля // Український нейрохірургічний журнал. - 2011. - № 1. - С. 60-65.

14. Щеколова, Н. Б. Возможности консервативной ортопедической коррекции постинсультной спастичности верхней конечности / Н. Б. Щеколова, А. М. Зиновьев // Пермский медицинский журнал. - 2017. - Том 34, № 2. - С. 15-19.

\section{References}

1. Aleksandrova A. Opyt raboty po razvitiju melkoj motoriki u detej s ONR. Doshkolnik. - Available at: http://doshkolnik.ru/motorika/10785-opyt-raboty.html. (accessed: 12.03.2017). (Russian).

2. Aretinskij VB, Telegina EV, Volkova LI. Recovery of motor function of the hand of stroke patients using the «Hand tutor». Ural Medical Journal. 2014;9(123):46-49. (Russian).

3. Volkova AM. Hirurgija kisti. Vol. 1, Hirurgija suhozhilij i nervov kisti. Ekaterinburg: Uralskij rabochij; 1991. 304

\section{p. (Russian).}

4. Lobzin YuV, Ivanova MV, Skripchenko NV, Pulman NF, Voitenkov VB, Naidin EYu. Experience of using Robotic Mechanotherapy in rehabilitation of children with motor disorders of various genesis. Medicine of Extreme Situations. 2015;1(51):22-26. (Russian).

5. Pirogova LA. Osnovy medicinskoj reabilitacii i nemedikamentoznoj terapii [Basics of medical rehabilitation and nonmedicamental therapy]. Grodno: GrGMU; 2008. 212 p. (Russian).

6. Baranov AA, Klochkova OA, Kurenkov AL, NamazovaBaranova LS, Nikitin SS, Artemenko AR, Mamedyarov AM. The role of brain plasticity in the functional adaptation of body at cerebral infantile paralysis with the affection of hands. Pediatric pharmacology. 2012;9(6):24-32. (Russian).

7. Smychek VB, Vasilchenko NI, Chapko IJa, Rjabceva TD. Osnovy medicinskoj reabilitacii pacientov, perenesshih mozgovoj insult. Nevrologija i nejrohirurgija. Vostochnaja Evropa [Neurology and neurosurgery. The Eastern Europe]. 2011;3:24-39. (Russian).

8. Smychek VB, Kopat TT, Glinskaya TH, Sushenko EF, Badulina LA. History and current state of medical and social assessment and rehabilitation system in Belarus Republic. Medical Journal. 2004;1(7):4-6. (Russian).

9. Shavlovskaja OA, inventor. Sposob ocenki sostojanija dvigatelnoj funkcii kisti [Method for estimating status of motor function of hand]. RU patent 2371088. 2009 Oct 27. (Russian).

10. Stoljarova LG, Tkacheva GR. Reabilitacija bolnyh s postinsultnymi dvigatelnymi rasstrojstvami. Moskva: Medicina; 1978. 216 p. (Russian).

11. Kodin AV, Lvov SE, Korobov NA, Rumjancev NA, Mironov DA, inventors. Ustrojstvo dlja biomehanicheskogo issledovanija kisti. RU patent 2066116. 1996 Sept 10. (Russian).

12. Borysov M, Danilchuk A, Varesnuk E, Skripka A, Litvinenko O. Physical rehabilitation of patients with amputating defects of upper limbs, in the period of training of using prostheses. Slobozhanskyi herald of science and sport. 2011;2(26):169-175. (Ukrainian).

13. Tsymbalyuk VI, Zozulya YuA. Selective fasciculotomy and neurostimulation in spasticity hand treatment. Ukrainian Neurosurgical Journal. 2011;1:60-65. (Ukrainian).

14. Schekolova NB, Zinoviev AM. Opportunities of conservative orthopedic correction of postinsult upper extremity spasticity. Perm Medical Journal. 2017;34(2):15-19. (Russian).

\title{
METHODS FOR EVALUATING FINE MOTOR SKILLS AND STRENGTH OF THE WRIST IN PATIENTS WITH CENTRAL AND PERIPHERAL PARESIS AND OPPORTUNITIES OF THEIR USE
}

\author{
But-Husaim U. U., Yarosh A. S.
}

Educational Institution «Grodno State Medical University», Grodno, Belarus

Literature and own data on the possibility of the study of the wrist strength and evaluation of fine motor skills in patients with central and peripheral paresis are presented and analyzed.

Keywords: Fine motor skills, wrist, paresis, evaluation of the effectiveness of medical rehabilitation 